\title{
Twisted Hochschild Homology of Quantum Flag Manifolds and Kähler Forms
}

\author{
Marco MATASSA \\ OsloMet - Oslo Metropolitan University, Oslo, Norway \\ E-mail: marco.matassa@oslomet.no
}

Received March 31, 2020, in final form September 25, 2020; Published online October 03, 2020

https://doi.org/10.3842/SIGMA.2020.098

\begin{abstract}
We study the twisted Hochschild homology of quantum flag manifolds, the twist being the modular automorphism of the Haar state. We prove that every quantum flag manifold admits a non-trivial class in degree two, with an explicit representative defined in terms of a certain projection. The corresponding classical two-form, via the HochschildKostant-Rosenberg theorem, is identified with a Kähler form on the flag manifold.
\end{abstract}

Key words: quantum flag manifolds; twisted Hochschild homology; Kähler forms

2020 Mathematics Subject Classification: 17B37; 20G42; 16E40

\section{Introduction}

The study of quantum homogeneous spaces corresponding to compact quantum groups is an active area of research. In this paper we study certain analogues of differential forms on quantum flag manifolds, which constitute a rich class of quantum homogeneous spaces.

Let us first explain how Hochschild homology fits into the picture. One possible approach to defining differential forms on non-commutative spaces is based on the Hochschild-KostantRosenberg theorem [14], or rather its continuous version. According to this theorem, the graded-commutative algebra of differential forms $\Omega^{\bullet}(M)$ on a smooth manifold $M$ is isomorphic to $H H_{\bullet}\left(C^{\infty}(M)\right)$, the Hochschild homology of the algebra $C^{\infty}(M)$ of smooth functions on $M$. Since $H_{\bullet}(A)$ makes sense for any associative algebra $A$, it is a candidate for the role of differential forms on the non-commutative space associated with $A$.

This works well for certain algebras, most notably the non-commutative tori [7], but it can be quite degenerate for other non-commutative spaces. In particular consider the compact quantum groups $\mathbb{C}_{q}[U]$, with $U$ a compact simple Lie group. Their Hochschild homology was computed by Feng and Tsygan in [9]. They find that the Hochschild homological dimension of $\mathbb{C}_{q}[U]$ is equal to $\operatorname{rank}(U)$, which is in stark constrast with that of $C^{\infty}(U)$ being $\operatorname{dim}(U)$. This phenomenon is usually referred to as a dimension drop.

Eventually it was discovered that the dimension drop for $\mathbb{C}_{q}[U]$ can be avoided by introducing an appropriate twisting in Hochschild homology. This was first observed by explicit computations for $\mathbb{C}_{q}\left[S U_{2}\right]$ in [11], and later elucidated by Brown and Zhang in [5]. They also show that this twisted Hochschild homology and the corresponding cohomology satisfy a general version of Poincaré duality, as described by Van den Bergh in [29]. The appropriate twisting turns out to be the modular automorphism of $\mathbb{C}_{q}[U]$, which we will denote by $\theta$, as shown by Dolgushev in the setting of Poisson geometry [8].

All of the above can be considered also for quantum flag manifolds. These are certain $*$-subalgebras of $\mathbb{C}_{q}[U]$ denoted by $\mathbb{C}_{q}\left[U / K_{S}\right]$, with $U / K_{S}$ a classical flag manifold. By the preceding

This paper is a contribution to the Special Issue on Noncommutative Manifolds and their Symmetries in honour of Giovanni Landi. The full collection is available at https://www.emis.de/journals/SIGMA/Landi.html 
discussion the twisted Hochschild homology $H H_{\bullet}^{\theta}\left(\mathbb{C}_{q}\left[U / K_{S}\right]\right)$ is an interesting object to study. This has been completely determined for the quantum 2-sphere in [10], but it is not known in general. In [23] we have studied the case of degree two for the quantum full flag manifolds $\mathbb{C}_{q}[U / T]$, with the result that $H H_{2}^{\theta}\left(\mathbb{C}_{q}[U / T]\right)$ has dimension at least $\operatorname{rank}(U)$, as well as providing explicit representatives for these classes.

An important motivation for studying the twisted Hochschild homology of $\mathbb{C}_{q}\left[U / K_{S}\right]$ in degree two is given by Kähler forms. Indeed, classically the flag manifolds $U / K_{S}$ are Kähler and hence admit these two-forms with special properties. The study of analogues of Kähler forms in the quantum setting is important from the point of view of non-commutative complex and Kähler geometry, see for instance [3, 24, 25].

We now come to the main results of this paper. Our first result shows the non-triviality of twisted Hochschild homology in degree two for any quantum flag manifold.

Theorem. Given any quantum flag manifold $\mathbb{C}_{q}\left[U / K_{S}\right]$, there exists a non-trivial class $[C(\mathrm{P})] \in$ $H H_{2}^{\theta}\left(\mathbb{C}_{q}\left[U / K_{S}\right]\right)$ represented by an explicit element $C(\mathrm{P}) \in \mathbb{C}_{q}\left[U / K_{S}\right]^{\otimes 3}$.

The explicit form involves a certain projection $\mathrm{P}$ with entries in $\mathbb{C}_{q}\left[U / K_{S}\right]$. Next, the representative $C(\mathrm{P})$ admits an appropriately defined classical limit for $q \rightarrow 1$. Under the HochschildKostant-Rosenberg theorem, this classical element corresponds to a two-form on $U / K_{S}$. Our second result shows that this is a Kähler form.

Theorem. Let $\omega_{\mathrm{P}} \in \Omega^{2}\left(U / K_{S}\right)$ be the form corresponding to $[C(\mathrm{P})]$ under the classical limit. Then $\omega_{\mathrm{P}}$ is a Kähler form on $U / K_{S}$.

We will actually construct the Kähler form $\omega_{\mathrm{P}}$ entirely in the classical setting, in a way which is suitable for comparison with the quantum case.

Finally let us mention the connection with another common approach to differential forms on quantum spaces, due to Woronowicz [30]. In this approach, given an algebra with an action of a compact quantum group, we introduce the structure of a differential calculus on the given algebra, together with various requirements about the action of the compact quantum group. In general we have many inequivalent choices of differential calculi, but the situation is much better for the quantum irreducible flag manifolds $\mathbb{C}_{q}\left[U / K_{S}\right]$. In this case it turns out that there is a unique analogue of the de Rham complex, which enjoys essentially all the classical properties, as shown by Heckenberger and Kolb in [13]. Within this setting, there is also a notion of Kähler forms introduced in [25]. Quantum irreducible flag manifolds were shown to admit Kähler forms in this sense in [22]. Finally we will show that these can also be identified with the forms $\omega_{P}$ in the classical limit.

The structure of the paper is as follows. In Section 2 we recall various preliminaries on Lie algebras and quantum groups and fix some notation. In Section 3 we summarize some results from [23] on twisted Hochschild homology of quantum flag manifolds. In Section 4 we prove that all quantum flag manifolds admit non-trivial classes in degree two. In Section 5 we introduce some Kähler forms on classical flag manifolds, from a point of view suitable for comparison with the quantum case. Finally, in Section 6 we compare the classical limit of the quantum classes with the Kähler forms obtained before.

\section{Notation and preliminaries}

In this section we fix most of our notation and recall various preliminary notions. These include Lie algebras and parabolic subalgebras on the classical side, and quantized enveloping algebras and coordinate rings on the quantum side. In particular we try to adopt notation that illustrate the link between the two sides as much as possible. 


\subsection{Lie algebras and Lie groups}

Let $\mathfrak{g}$ be a complex simple Lie algebra with fixed Cartan subalgebra $\mathfrak{h}$. We denote by $\Delta(\mathfrak{g})$ the roots of $\mathfrak{g}$ with respect to $\mathfrak{h}$, and by $\Delta^{ \pm}(\mathfrak{g})$ a choice of positive/negative roots. We write $\left\{\alpha_{i}: i \in I\right\}$ for the simple roots and $\left\{\omega_{i}: i \in I\right\}$ for the fundamental weights. We denote by $(\cdot, \cdot)$ the bilinear form on $\mathfrak{h}^{*}$ obtained from the Killing form, rescaled in such a way that $(\alpha, \alpha)=2$ for the short roots.

Given $\alpha \in \Delta^{+}(\mathfrak{g})$, we choose root vectors $e_{\alpha} \in \mathfrak{g}_{\alpha}$ and $f_{\alpha} \in \mathfrak{g}_{-\alpha}$ normalized as in [19, Theorem 6.6]. In particular we have $\left[e_{\alpha}, f_{\alpha}\right]=h_{\alpha}$ and $\alpha\left(h_{\beta}\right)=(\alpha, \beta)$.

We denote by $\mathfrak{u}$ the compact real form of $\mathfrak{g}$. It is given by

$$
\mathfrak{u}=\bigoplus_{i \in I} \mathbb{R} \boldsymbol{i} h_{\alpha_{i}} \oplus \bigoplus_{\alpha \in \Delta^{+}(\mathfrak{g})} \mathbb{R}\left(e_{\alpha}-f_{\alpha}\right) \oplus \bigoplus_{\alpha \in \Delta^{+}(\mathfrak{g})} \mathbb{R} \boldsymbol{i}\left(e_{\alpha}+f_{\alpha}\right)
$$

Here and in the following $\boldsymbol{i} \in \mathbb{C}$ will denote the imaginary unit.

Corresponding to the Lie algebras $\mathfrak{g}$ and $\mathfrak{u}$, we have the connected, simply-connected Lie groups $G$ and $U$. We will also denote by $T$ the maximal torus of $U$.

\subsection{Parabolic subalgebras and flag manifolds}

Let $S$ be a subset of the simple roots, that is $S \subset I$. Corresponding to this choice, we define the roots

$$
\Delta\left(\mathfrak{l}_{S}\right):=\operatorname{span}\left\{\alpha_{i}: i \in S\right\} \cap \Delta(\mathfrak{g}) .
$$

In terms of these we define the Levi factor corresponding to $S$ by

$$
\mathfrak{l}_{S}:=\mathfrak{h} \oplus \bigoplus_{\alpha \in \Delta\left(\mathfrak{l}_{S}\right)} \mathfrak{g}_{\alpha}
$$

It is a reductive Lie subalgebra containing the Cartan subalgebra. We also define

$$
\Delta\left(\mathfrak{n}_{S}^{ \pm}\right):=\Delta^{ \pm}(\mathfrak{g}) \backslash \Delta^{ \pm}\left(\mathfrak{l}_{S}\right)
$$

In other words, the set $\Delta\left(\mathfrak{n}_{S}^{+}\right)$contains the positive roots which are not in $\Delta\left(\mathfrak{l}_{S}\right)$, and similarly for $\Delta\left(\mathfrak{n}_{S}^{-}\right)$. In terms of these roots we define

$$
\mathfrak{n}_{S}^{ \pm}:=\bigoplus_{\alpha \in \Delta\left(\mathfrak{n}_{S}^{ \pm}\right)} \mathfrak{g}_{\alpha}
$$

These are also Lie subalgebras of $\mathfrak{g}$, which we call the positive and negative nilradical corresponding to $S$. They are $\mathfrak{l}_{S}$-modules with respect to the adjoint action.

In terms of the previously defined subalgebras we have the decomposition

$$
\mathfrak{g}=\mathfrak{n}_{S}^{+} \oplus \mathfrak{l}_{S} \oplus \mathfrak{n}_{S}^{-}
$$

We call $\mathfrak{p}_{S}:=\mathfrak{l}_{S} \oplus \mathfrak{n}_{S}^{+}$the standard parabolic subalgebra corresponding to $S$. Here standard parabolic means that $\mathfrak{p}_{S}$ is a subalgebra containing the standard Borel subalgebra

$$
\mathfrak{b}:=\mathfrak{h} \oplus \bigoplus_{\alpha \in \Delta^{+}(\mathfrak{g})} \mathfrak{g}_{\alpha}
$$

In the case $S=\varnothing$ we have $\mathfrak{p}_{S}=\mathfrak{b}$. We also note that $\mathfrak{g} / \mathfrak{p}_{S} \cong \mathfrak{n}_{S}^{-}$as $\mathfrak{l}_{S}$-modules. 
In terms of the compact real form $\mathfrak{u}$, we will consider

$$
\mathfrak{k}_{S}:=\mathfrak{l}_{S} \cap \mathfrak{u}, \quad \mathfrak{m}_{S}:=\left(\mathfrak{n}_{S}^{+} \oplus \mathfrak{n}_{S}^{-}\right) \cap \mathfrak{u} .
$$

We have that $\mathfrak{k}_{S}$ is a Lie subalgebra of $\mathfrak{u}$, but this is not the case for $\mathfrak{m}_{S}$.

Corresponding to these Lie subalgebras, we have the (generalized) flag manifolds. These are homogeneous spaces of the form $G / P_{S}$, where $P_{S}$ is the parabolic subgroup with Lie algebra $\mathfrak{p}_{S}$. This definition shows that they are complex manifolds. It is also possible to give a realization in terms of $U$, the compact real form of $G$, by the diffeomorphism

$$
G / P_{S} \cong U / K_{S}, \quad K_{S}:=P_{S} \cap U
$$

The realization $U / K_{S}$ makes it clear that they are compact manifolds. Finally there is also a projective realization of the flag manifolds, which we will describe in Section 4.

Some notable subclasses are the following. For $S=\varnothing$ we have the homogeneous spaces $G / B \cong U / T$, called the full flag manifolds. At the other extreme we have the irreducible flag manifolds, corresponding to the case $S=I \backslash\{t\}$ with $\alpha_{t}$ having multiplicity 1 in the highest root of $\mathfrak{g}$. These include the Grassmannians, for instance.

\subsection{Quantized enveloping algebras}

We will use the conventions of [18], which will be our main reference for this part. Given $0<q<1$, the quantized enveloping algebra $U_{q}(\mathfrak{g})$ is a Hopf algebra deformation of the enveloping algebra $U(\mathfrak{g})$ defined as follows. It has generators $\left\{K_{i}, E_{i}, F_{i}\right\}_{i=1}^{r}$, with $r:=\operatorname{rank}(\mathfrak{g})$, and relations as in [18, Section 6.1.2]. In particular, the comultiplication, antipode and counit are given by

$$
\begin{array}{lll}
\Delta\left(K_{i}\right)=K_{i} \otimes K_{i}, & \Delta\left(E_{i}\right)=E_{i} \otimes K_{i}+1 \otimes E_{i}, & \Delta\left(F_{i}\right)=F_{i} \otimes 1+K_{i}^{-1} \otimes F_{i}, \\
S\left(K_{i}\right)=K_{i}^{-1}, & S\left(E_{i}\right)=-E_{i} K_{i}^{-1}, & S\left(F_{i}\right)=-K_{i} F_{i}, \\
\varepsilon\left(K_{i}\right)=1, & \varepsilon\left(E_{i}\right)=0, & \varepsilon\left(F_{i}\right)=0 .
\end{array}
$$

Given $\lambda=\sum_{i=1}^{r} n_{i} \alpha_{i}$ we will write $K_{\lambda}:=K_{1}^{n_{1}} \cdots K_{r}^{n_{r}}$. Let $\rho:=\frac{1}{2} \sum_{\alpha>0} \alpha$ be the half-sum of the positive roots of $\mathfrak{g}$. Then we have $S^{2}(X)=K_{2 \rho} X K_{2 \rho}^{-1}$ for any $X \in U_{q}(\mathfrak{g})$.

We will also consider a $*$-structure on $U_{q}(\mathfrak{g})$, which in the classical case corresponds to the compact real form $\mathfrak{u}$. We can take for instance

$$
K_{i}^{*}=K_{i}, \quad E_{i}^{*}=K_{i} F_{i}, \quad F_{i}^{*}=E_{i} K_{i}^{-1} .
$$

The precise formulae are not so important here, as any equivalent $*$-structure will work equally well for our purposes. We will write $U_{q}(\mathfrak{u}):=\left(U_{q}(\mathfrak{g}), *\right)$ when we consider $U_{q}(\mathfrak{g})$ endowed with the $*$-structure corresponding to the compact real form.

We will also consider a quantum analogue of the Levi factor $\mathfrak{l}_{S}$, following [28]. The quantized Levi factor $U_{q}\left(\mathfrak{l}_{S}\right)$ is defined by

$$
U_{q}\left(\mathfrak{l}_{S}\right):=\left\langle K_{i}, E_{j}, F_{j}: i \in I, j \in S\right\rangle \subset U_{q}(\mathfrak{g}) .
$$

Here $\langle\cdot\rangle$ denotes the subalgebra generated by the given elements in $U_{q}(\mathfrak{g})$. It is easily verified that $U_{q}\left(\mathfrak{l}_{S}\right)$ is a Hopf subalgebra. Moreover it is a Hopf $*$-subalgebra with $*$ corresponding to the compact real form. Taking the $*$-structure into account we write $U_{q}\left(\mathfrak{k}_{S}\right):=\left(U_{q}\left(\mathfrak{l}_{S}\right), *\right)$. In the special case $S=\varnothing$ we write instead $U_{q}(\mathfrak{t}):=\left\langle K_{i}: i \in I\right\rangle$ with its $*$-structure. 


\subsection{Quantized coordinate rings}

The quantized coordinate ring $\mathbb{C}_{q}[G]$ is defined as a subspace of the linear dual $U_{q}(\mathfrak{g})^{*}$. We take the span of all the matrix coefficients of the finite-dimensional irreducible representations $V(\lambda)$ (see below). It becomes a Hopf algebra by duality in the following manner: given $X, Y \in U_{q}(\mathfrak{g})$ and $a, b \in \mathbb{C}_{q}[G]$ we define

$$
\begin{aligned}
& (a b)(X):=(a \otimes b) \Delta(X), \quad \Delta(a)(X \otimes Y):=a(X Y), \\
& S(a)(X):=a(S(X)), \quad 1(X):=\varepsilon(X), \quad \varepsilon(a):=a(1) .
\end{aligned}
$$

Moreover it becomes a Hopf $*$-algebra by setting

$$
a^{*}(X):=\overline{a\left(S(X)^{*}\right)} .
$$

We write $\mathbb{C}_{q}[U]:=\left(\mathbb{C}_{q}[G], *\right)$ for $\mathbb{C}_{q}[G]$ endowed with this $*$-structure.

We have a left action $\triangleright$ and a right action $\triangleleft$ of $U_{q}(\mathfrak{g})$ on $\mathbb{C}_{q}[G]$ given by

$$
(X \triangleright a)(Y):=a(Y X), \quad(a \triangleleft X)(Y):=a(X Y) .
$$

Using the action of $U_{q}(\mathfrak{g})$ on $\mathbb{C}_{q}[G]$ we can define quantum analogues of the (generalized) flag manifolds. The quantum flag manifold $\mathbb{C}_{q}\left[U / K_{S}\right]$ is defined by

$$
\mathbb{C}_{q}\left[U / K_{S}\right]:=\left\{a \in \mathbb{C}_{q}[U]: X \triangleright a=\varepsilon(X) a, \forall X \in U_{q}\left(\mathfrak{k}_{S}\right)\right\} .
$$

Notice that $\mathbb{C}_{q}\left[U / K_{S}\right]=\mathbb{C}_{q}[U]^{U_{q}\left(\mathfrak{k}_{S}\right)}$, the invariants with respect to the action of $U_{q}\left(\mathfrak{k}_{S}\right)$. In the special case $S=\varnothing$ we will write $\mathbb{C}_{q}[U / T]:=\mathbb{C}_{q}[U]^{U_{q}(\mathfrak{t})}$, as this case corresponds to the quantum analogue of the full flag manifold $U / T$.

\subsection{Matrix coefficients}

The representation theory of $U_{q}(\mathfrak{g})$ is essentially the same as that of $U(\mathfrak{g})$, hence of $\mathfrak{g}$. In particular we have the analogue of the highest weight modules $V(\lambda)$ for any dominant weight $\lambda$, which we will denote by the same symbol. In any case, given a finite-dimensional representation $V$, we define its matrix coefficients by

$$
\left(c_{f, v}^{V}\right)(X):=f(X v), \quad f \in V^{*}, \quad v \in V, \quad X \in U_{q}(\mathfrak{g}) .
$$

These elements span $\mathbb{C}_{q}[G]$, according to the description given above.

In particular we will be interested in unitary representations. We say that an inner product $(\cdot, \cdot)$ on $V$ is $U_{q}(\mathfrak{u})$-invariant if it satisfies

$$
(X v, w)=\left(v, X^{*} w\right), \quad \forall v, w \in V, \quad \forall X \in U_{q}(\mathfrak{u}) .
$$

Here we use the $*$-structure of $U_{q}(\mathfrak{u})$. It is well-known that an $U_{q}(\mathfrak{u})$-invariant inner product exists on every representation $V(\lambda)$, and it is unique up to a constant. Let $\left\{v_{i}\right\}_{i}$ be an orthonormal weight basis of $V(\lambda)$ with respect to $(\cdot, \cdot)$, and write $\lambda_{i}$ for the weight of $v_{i}$. Also denote by $\left\{f^{i}\right\}_{i}$ the dual basis of $V(\lambda)^{*}$. With this notation we set

$$
\left(u^{V(\lambda)}\right)_{j}^{i}:=C_{f^{i}, v_{j}}^{V(\lambda)}
$$

We will often omit the superscript $V(\lambda)$ from the notation, as we will mostly work with one fixed representation in the following. Note that we have

$$
u_{j}^{i}(X)=f^{i}\left(X v_{j}\right)=\left(v_{i}, X v_{j}\right) .
$$




\subsection{Modular automorphism}

The Hopf algebras $\mathbb{C}_{q}[U]$ (or rather their completions as $C^{*}$-algebras) are examples of compact quantum groups. According to the general theory, there is a unique state $h: \mathbb{C}_{q}[U] \rightarrow \mathbb{C}$, called the Haar state, satisfying the properties

$$
(h \otimes \mathrm{id}) \circ \Delta(a)=h(a)=(\mathrm{id} \otimes h) \circ \Delta(a), \quad \forall a \in \mathbb{C}_{q}[U] .
$$

This state is not a trace, but instead we have the property

$$
h(a b)=h(b \theta(a)), \quad \forall a, b \in \mathbb{C}_{q}[U],
$$

where $\theta$ is the modular automorphism corresponding to the Haar state.

The modular automorphism has a simple expression in terms of the action of $U_{q}(\mathfrak{u})$ on $\mathbb{C}_{q}[U]$. Recall that $S^{2}(X)=K_{2 \rho} X K_{2 \rho}^{-1}$ for all $X \in U_{q}(\mathfrak{u})$, from which it follows that $S^{2}(a)=K_{2 \rho}^{-1} \triangleright a \triangleleft K_{2 \rho}$ for all $a \in \mathbb{C}_{q}[U]$. By [18, Chapter 11, Proposition 34] we have

$$
\theta(a)=K_{2 \rho} \triangleright a \triangleleft K_{2 \rho}, \quad \forall a \in \mathbb{C}_{q}[U]
$$

In particular, for the unitary matrix coefficients $u_{j}^{i}$ we have

$$
\theta\left(u_{j}^{i}\right)=q^{\left(2 \rho, \lambda_{i}+\lambda_{j}\right)} u_{j}^{i},
$$

where $\lambda_{i}$ denotes the weight of the basis element $v_{i}$.

\section{Some results on twisted Hochschild homology}

In this section we recall some basics of (twisted) Hochschild homology, as well as some results obtained in [23]. In particular we discuss how to construct certain 2-cycles on quantum flag manifolds in terms of appropriate projections.

\section{1 (Twisted) Hochschild homology}

Hochschild homology is a homology theory for associative algebras, which we consider here to be over $\mathbb{C}$. The main reference for this section is [21]. Let $A$ be an associative algebra and $M$ be an $A$-bimodule. Write $C_{n}(A, M):=M \otimes A^{\otimes n}$. The Hochschild boundary is the linear map $\mathrm{b}: C_{n}(A, M) \rightarrow C_{n-1}(A, M)$ given by

$$
\begin{aligned}
\mathrm{b}\left(m \otimes a_{1} \otimes \cdots \otimes a_{n}\right):= & m a_{1} \otimes \cdots \otimes a_{n}+\sum_{i=1}^{n-1}(-1)^{i} m \otimes a_{1} \otimes \cdots \otimes a_{i} a_{i+1} \otimes \cdots \otimes a_{n} \\
& +(-1)^{n} a_{n} m \otimes a_{1} \otimes \cdots \otimes a_{n-1} .
\end{aligned}
$$

It satisfies $\mathrm{b}^{2}=0$, hence we have corresponding homology groups denoted by $H_{\bullet}(A, M)$. We will also use the notation $H_{\bullet}(A):=H_{\bullet}(A, A)$. Hochschild homology can also be defined in terms of derived functors as $H_{n}(A, M)=\operatorname{Tor}_{n}^{A^{e}}(A, M)$, where $A^{e}:=A \otimes A^{\mathrm{op}}$. There is a corresponding dual cohomology theory, whose groups are denoted by $H^{n}(A, M)$.

A natural choice of bimodule is given by $M=A$, in which case we talk about the Hochschild homology of $A$. Here we will focus on the twisted bimodules $M={ }_{\sigma} A$, which are defined as follows: as a vector space we have $M=A$, but the bimodule structure is given by $a \cdot b \cdot c=\sigma(a) b c$, where $\sigma \in \operatorname{Aut}(A)$. In this case we will use the notation $H H_{\bullet}^{\sigma}(A):=H_{\bullet}\left(A,{ }_{\sigma} A\right)$ and refer to it as the twisted Hochschild homology of $A$ (with twist $\sigma$ ). Notice that we could also introduce a twist for the right multiplication, but as bimodules this gives nothing new. 
Remark 3.1. The definition of twisted Hochschild homology $H H_{\bullet}^{\sigma}(A)$ presented here is essentially as in [11], while the original cohomological setting appeared first in [20]. There is also a relation with the notion of braided Hochschild homology introduced in [1] and [2], for a discussion of these matters see [12, Example 3.9].

In the case when $A$ is the algebra of functions on some smooth space $X$, the Hochschild homo$\log y H_{\bullet}(A)$ of $A$ is related to the differential forms on the space $X$. This is the HochschildKostant-Rosenberg theorem [14], see also [21, Theorem 3.4.4]. Recall that for a commutative unital algebra $A$, we have the $A$-module of differential forms $\Omega^{\bullet}(A):=\bigwedge_{A}^{\bullet} \Omega^{1}(A)$ constructed from the module of Kähler differentials $\Omega^{1}(A)$.

Theorem (Hochschild-Kostant-Rosenberg). Let $A$ be a commutative smooth algebra over $\mathbb{C}$. Then there is an isomorphism of graded $\mathbb{C}$-algebras $H_{\bullet}(A) \cong \Omega^{\bullet}(A)$.

For the notion of smooth algebra see [21, Section 3.4.1]. The algebra structure on $H_{\bullet}(A)$ is given by the shuffle product, which relies on commutativity of $A$, see [21, Section 4.2]. There is also a continuous version of this theorem, essentially due to Connes [7], which allows to consider smooth forms as opposed to algebraic ones.

Finally we note that, at the level of chains, the map $A^{\otimes n+1} \rightarrow \Omega^{n}(A)$ is given by

$$
a_{0} \otimes a_{1} \otimes \cdots a_{n} \mapsto a_{0} \mathrm{~d} a_{1} \wedge \cdots \wedge \mathrm{d} a_{n} .
$$

\subsection{Some results}

We will now focus on the quantum flag manifolds $\mathbb{C}_{q}\left[U / K_{S}\right]$. We will recall some results on their twisted Hochschild homology and cohomology from [23].

Fix an irreducible representation $V(\lambda)$ of $U_{q}(\mathfrak{u})$ and write $N:=\operatorname{dim} V(\lambda)$. Let $\left\{v_{i}\right\}_{i=1}^{N}$ be an orthonormal weight basis with respect to an $U_{q}(\mathfrak{u})$-invariant inner product, and write $\lambda_{i}$ for the weight of $v_{i}$. Denote by $u_{j}^{i}=\left(u^{V(\lambda)}\right)_{j}^{i}$ the unitary matrix coefficients.

Denote by $\operatorname{Mat}\left(\mathbb{C}_{q}[U]\right)$ the set of all matrices with entries in $\mathbb{C}_{q}[U]$. Given $a, b \in\{1, \ldots, N\}$, we define the $N \times N$-matrix $\mathrm{M}_{b}^{a} \in \operatorname{Mat}\left(\mathbb{C}_{q}[U]\right)$ with entries

$$
\left(\mathrm{M}_{a}^{b}\right)_{j}^{i}:=u_{a}^{i}\left(u_{b}^{j}\right)^{*}, \quad i, j \in\{1, \ldots, N\}
$$

The $*$-structure is extended to matrices by the conjugate transpose, that is $\left(M^{*}\right)_{j}^{i}:=\left(M_{i}^{j}\right)^{*}$ for $M \in \operatorname{Mat}\left(\mathbb{C}_{q}[U]\right)$. We also define the quantum trace by

$$
\operatorname{Tr}_{q}\left(\mathrm{M}_{a}^{b}\right):=\sum_{i=1}^{N} q^{\left(2 \rho, \lambda_{i}\right)}\left(\mathrm{M}_{a}^{b}\right)_{i}^{i}
$$

The matrices $\mathrm{M}_{a}^{b}$ behave like matrix units, as shown in [23, Proposition 3.3].

Proposition 3.2. The matrices $\left\{\mathrm{M}_{a}^{b}\right\}_{a, b}$ are linearly independent and satisfy

$$
\left(\mathrm{M}_{b}^{a}\right)^{*}=\mathrm{M}_{a}^{b}, \quad \mathrm{M}_{b}^{a} \mathrm{M}_{d}^{c}=\delta_{d}^{a} \mathrm{M}_{b}^{c}, \quad \operatorname{Tr}_{q}\left(\mathrm{M}_{b}^{a}\right)=\delta_{b}^{a} q^{\left(2 \rho, \lambda_{a}\right)} .
$$

Remark 3.3. These matrices are denoted by $\mathrm{N}_{a}^{b}$ in [23]. In the cited paper we also considered the matrices $\mathrm{M}_{a}^{b}$ given by $\left(u_{i}^{m}\right)^{*} u_{j}^{n}$, but we will not use them here.

In particular, the elements $\mathrm{P}_{a}:=\mathrm{M}_{a}^{a}$ are self-adjoint projections of "quantum rank one". We will use them to construct certain twisted Hochschild 2-cycles on $\mathbb{C}_{q}[U]$.

Remark 3.4. It is worth noting that we have $\mathrm{P}_{a} \in \operatorname{Mat}\left(\mathbb{C}_{q}[U / T]\right)$, essentially by construction. On the other hand, we need additional conditions to get $\mathrm{P}_{a} \in \operatorname{Mat}\left(\mathbb{C}_{q}\left[U / K_{S}\right]\right)$. 
Let us consider the elements $C\left(\mathrm{P}_{a}\right) \in \mathbb{C}_{q}[U]^{\otimes 3}$ defined by

$$
C\left(\mathrm{P}_{a}\right):=\operatorname{Tr}_{q}\left(\left(2 \mathrm{P}_{a}-1\right) \otimes \mathrm{P}_{a} \otimes \mathrm{P}_{a}\right) .
$$

Here the quantum trace is extended in the obvious way, namely

$$
C\left(\mathrm{P}_{a}\right)=\sum_{i, j, k=1}^{N} q^{\left(2 \rho, \lambda_{i}\right)}\left(2 \mathrm{P}_{a}-1\right)_{j}^{i} \otimes\left(\mathrm{P}_{a}\right)_{k}^{j} \otimes\left(\mathrm{P}_{a}\right)_{i}^{k} .
$$

Recall that $\theta$ denotes the modular automorphism of $\mathbb{C}_{q}[U]$, which acts by $\theta\left(u_{j}^{i}\right)=q^{\left(2 \rho, \lambda_{i}+\lambda_{j}\right)} u_{j}^{i}$. The following result can be found in [23, Proposition 5.1].

Proposition 3.5. The element $C\left(\mathrm{P}_{a}\right) \in \mathbb{C}_{q}[U]^{\otimes 3}$ is a 2-cycle in the (normalized) twisted Hochschild complex, hence it defines a class

$$
\left[C\left(\mathrm{P}_{a}\right)\right] \in H H_{2}^{\theta}\left(\mathbb{C}_{q}[U]\right) .
$$

Moreover, if $\left(\mathrm{P}_{a}\right)_{j}^{i} \in \mathbb{C}_{q}\left[U / K_{S}\right]$ for all entries, then we also have a class

$$
\left[C\left(\mathrm{P}_{a}\right)\right] \in H H_{2}^{\theta}\left(\mathbb{C}_{q}\left[U / K_{S}\right]\right) .
$$

Remark 3.6. Here $C\left(\mathrm{P}_{a}\right)$ is a modification of the usual Chern character $\mathrm{Ch}_{n}: K_{0}(A) \rightarrow H_{2 n}^{\lambda}(A)$ given by $\mathrm{Ch}_{n}(P)=\operatorname{Tr}\left(P^{\otimes 2 n+1}\right)$, where $H_{\bullet}^{\lambda}$ denotes the cyclic homology of $A$. This can be easily modified to the twisted case by using the quantum trace, as opposed to the usual trace. On the other hand, the factor $2 \mathrm{P}_{a}-1$ and the property $\operatorname{Tr}_{q}\left(\mathrm{P}_{a}\right)=q^{\left(2 \rho, \lambda_{a}\right)}$ guarantee that we map lands in Hochschild homology, as opposed to cyclic homology.

Next we would like to check whether the class $\left[C\left(\mathrm{P}_{a}\right)\right]$ is non-trivial. To do this we can introduce an appropriate cohomology class and to show that the corresponding pairing is non-zero. Given $a \in I$, consider the linear functional $\eta_{a}: \mathbb{C}_{q}[U]^{\otimes 3} \rightarrow \mathbb{C}$ given by

$$
\eta_{a}\left(a_{0} \otimes a_{1} \otimes a_{2}\right):=\varepsilon\left(a_{0}\right) \varepsilon\left(F_{a} \triangleright a_{1}\right) \varepsilon\left(E_{a} \triangleright a_{2}\right) .
$$

It is easy to check that, due to the properties of the counit, these linear functionals define (twisted) cohomology classes, as shown in [23, Proposition 5.5].

Proposition 3.7. The restriction of $\eta_{a}$ to $\mathbb{C}_{q}\left[U / K_{S}\right]$ gives a cohomology class

$$
\left[\eta_{a}\right] \in H H_{\theta}^{2}\left(\mathbb{C}_{q}\left[U / K_{S}\right]\right) .
$$

Remark 3.8. Observe that this is true for the restriction of $\eta_{a}$ to any quantum flag manifold $\mathbb{C}_{q}\left[U / K_{S}\right]$. On the other hand, these functionals do not give classes in $H H_{\theta}^{2}\left(\mathbb{C}_{q}[U]\right)$.

Finally we look at the pairing between $\left[\eta_{a}\right]$ and $\left[C\left(\mathrm{P}_{b}\right)\right]$. The result can be expressed entirely in terms of representation-theoretic data, as shown in [23, Proposition 6.11].

Proposition 3.9. The pairing between $\left[\eta_{a}\right]$ and $\left[C\left(\mathrm{P}_{b}\right)\right]$ is given by

$$
\left\langle\left[\eta_{a}\right],\left[C\left(\mathrm{P}_{b}\right)\right]\right\rangle=q^{\left(2 \rho-\alpha_{a}, \lambda_{b}\right)}\left[\left(\alpha_{a}^{\vee}, \lambda_{b}\right)\right]_{q_{a}} .
$$

Here $\alpha^{\vee}=2 \alpha /(\alpha, \alpha)$ is the coroot corresponding to $\alpha$.

Our aim in the next section will be to produce some non-trivial classes in $H H_{2}^{\theta}\left(\mathbb{C}_{q}\left[U / K_{S}\right]\right)$. In order to do this, we will proceed in two steps:

1) define a projection $\mathrm{P}_{1} \in \operatorname{Mat}\left(\mathbb{C}_{q}\left[U / K_{S}\right]\right)$, giving a class $\left[C\left(\mathrm{P}_{1}\right)\right] \in H H_{2}^{\theta}\left(\mathbb{C}_{q}\left[U / K_{S}\right]\right)$,

2) prove that it is non-trivial by showing that $\left\langle\left[\eta_{a}\right],\left[C\left(\mathrm{P}_{1}\right)\right]\right\rangle \neq 0$ for some $a \in I$. 


\section{Non-trivial classes on quantum flag manifolds}

In this section we will construct some non-trivial classes in $H H_{2}^{\theta}\left(\mathbb{C}_{q}\left[U / K_{S}\right]\right)$. The first step will be to construct appropriate projections $\mathrm{P}_{1} \in \operatorname{Mat}\left(\mathbb{C}_{q}\left[U / K_{S}\right]\right)$. This will make use of a certain irreducible representation, which in the classical case is used to give a projective realization of $U / K_{S}$. With these projections at hand and the results of the previous section, it will be fairly straightforward to show that we get non-trivial classes.

\subsection{The projections}

It is well-known that the flag manifold $U / K_{S}$ can be realized as a $U$-orbit in a projective space, see for instance [6, Section 3.2.8]. The projective space here is $\mathbb{P}\left(V\left(\rho_{S}\right)\right)$, where $V\left(\rho_{S}\right)$ is the irreducible representation with highest weight

$$
\rho_{S}:=\sum_{i \in I \backslash S} \omega_{i}
$$

In the quantum case we proceed along these lines by considering the corresponding irreducible representation $V\left(\rho_{S}\right)$ of $U_{q}(\mathfrak{u})$. Let $\left\{v_{i}\right\}_{i}$ be an orthonormal weight basis of $V\left(\rho_{S}\right)$ with respect to a $U_{q}(\mathfrak{u})$-invariant inner product. We write $u_{j}^{i}=\left(u^{V\left(\rho_{S}\right)}\right)_{j}^{i}$ for the unitary matrix coefficients. For notational convenience, we will assume from now on that $v_{1}$ is a highest weight vector of $V\left(\rho_{S}\right)$, hence of corresponding weight $\lambda_{1}=\rho_{S}$.

With the notation as above, we define the elements

$$
p_{j}^{i}:=u_{1}^{i}\left(u_{1}^{j}\right)^{*} \in \mathbb{C}_{q}[U] .
$$

Notice that $\left(\mathrm{P}_{1}\right)_{j}^{i}=p_{j}^{i}$, using the notation of the previous section. Our goal will be to show that $p_{j}^{i} \in \mathbb{C}_{q}\left[U / K_{S}\right]$. First we will need the following lemma.

Lemma 4.1. We have $F_{i} v_{1}=0$ for all $i \in S$.

Proof. This works as in the classical case, but we provide a proof for completeness. Suppose that $F_{i} v_{1} \neq 0$, which implies that $F_{i} v_{1}$ has weight $\rho_{S}-\alpha_{i}$. Recall that the Weyl group acts transitively on the weights of an irreducible representation. Denoting by $s_{\alpha}(\lambda)=\lambda-\frac{2(\lambda, \alpha)}{(\alpha, \alpha)} \alpha$ the reflection of $\lambda$ with respect to $\alpha$, we find that $s_{\alpha_{i}}\left(\rho_{S}-\alpha_{i}\right)=s_{\alpha_{i}}\left(\rho_{S}\right)+\alpha_{i}$. Moreover, since $\left(\rho_{S}, \alpha_{i}\right)=0$ for $i \in S$ by definition of $\rho_{S}$, we obtain $s_{\alpha_{i}}\left(\rho_{S}-\alpha_{i}\right)=\rho_{S}+\alpha_{i}$. But this is impossible, since $\rho_{S}$ is the highest weight, hence we must have $F_{i} v_{1}=0$.

We are now ready to construct the invariant projections.

Proposition 4.2. We have $p_{j}^{i} \in \mathbb{C}_{q}\left[U / K_{S}\right]$. Moreover we have

$$
\sum_{k} p_{k}^{i} p_{j}^{k}=p_{j}^{i}, \quad\left(p_{j}^{i}\right)^{*}=p_{i}^{j}, \quad \sum_{i} q^{\left(2 \rho, \lambda_{i}\right)} p_{i}^{i}=q^{\left(2 \rho, \rho_{S}\right)}
$$

Proof. Since $p_{j}^{i}=\left(\mathrm{M}_{1}^{1}\right)_{j}^{i}$, all claims follow from Proposition 3.2 except for $p_{j}^{i} \in \mathbb{C}_{q}\left[U / K_{S}\right]$. For this it suffices to show that $p_{j}^{i}$ is invariant under the generators of $U_{q}\left(\mathfrak{k}_{S}\right)$.

Since $\left(X \triangleright u_{1}^{i}\right)(Y)=\left(v_{i}, Y X v_{1}\right)$, it is clear that $E_{k} \triangleright u_{1}^{i}=0$ for $k \in S$ (this is true for any $k$, since $v_{1}$ is a highest weight vector). Next we have $F_{k} \triangleright u_{1}^{i}=0$ for $k \in S$, by Lemma 4.1. On the other hand we have $K_{k} \triangleright u_{1}^{i}=q^{\left(\rho_{S}, \alpha_{k}\right)} u_{1}^{i}$. Observe that $q^{\left(\rho_{S}, \alpha_{k}\right)} \neq 1$ for $k \notin S$.

Now consider $\left(u_{1}^{j}\right)^{*}$. Since $\left(u_{1}^{j}\right)^{*}(X)=\overline{\left(v_{j}, S(X)^{*} v_{1}\right)}$ we have

$$
\left(X \triangleright\left(u_{1}^{j}\right)^{*}\right)(Y)=\overline{\left(v_{j}, S(Y X)^{*} v_{1}\right)}=\overline{\left(v_{j}, S(Y)^{*} S(X)^{*} v_{1}\right)} .
$$


We easily conclude that $E_{k} \triangleright\left(u_{1}^{j}\right)^{*}=F_{k} \triangleright\left(u_{1}^{j}\right)^{*}=0$ for $k \in S$, as for the elements $u_{1}^{i}$. On the other hand we have $K_{i} \triangleright\left(u_{1}^{j}\right)^{*}=q^{-\left(\rho_{S}, \alpha_{k}\right)}\left(u_{1}^{j}\right)^{*}$, since $S\left(K_{k}\right)^{*}=K_{k}^{-1}$.

Finally consider the elements $p_{j}^{i}$. Using $X \triangleright(a b)=\left(X_{(1)} \triangleright a\right)\left(X_{(2)} \triangleright b\right)$ it is clear that

$$
E_{k} \triangleright p_{j}^{i}=0, \quad F_{k} \triangleright p_{j}^{i}=0, \quad k \in S .
$$

On the other hand, using the results above, we have for any $k \in I$ that

$$
K_{k} \triangleright p_{j}^{i}=\left(K_{k} \triangleright u_{1}^{i}\right)\left(K_{k} \triangleright\left(u_{1}^{j}\right)^{*}\right)=p_{j}^{i} .
$$

Since $X \triangleright p_{j}^{i}=\varepsilon(X) p_{j}^{i}$ for the generators of $U_{q}\left(\mathfrak{k}_{S}\right)$, we have $p_{j}^{i} \in \mathbb{C}_{q}\left[U / K_{S}\right]$.

Remark 4.3. It can be shown that the elements $p_{j}^{i}$ generate the quantum flag manifold $\mathbb{C}_{q}\left[U / K_{S}\right]$, see $[13$, Proposition 3.2$]$, but we will not need this fact.

\subsection{Non-triviality}

We have just constructed a projection $\mathrm{P}_{1}$ with entries

$$
\left(\mathrm{P}_{1}\right)_{j}^{i}=p_{j}^{i}=u_{1}^{i}\left(u_{1}^{j}\right)^{*} \in \mathbb{C}_{q}\left[U / K_{S}\right] .
$$

By Propositions 3.5 and 4.2, we have a corresponding class

$$
\left[C\left(\mathrm{P}_{1}\right)\right] \in H H_{2}^{\theta}\left(\mathbb{C}_{q}\left[U / K_{S}\right]\right) .
$$

Finally we want to show that this class is non-trivial.

Theorem 4.4. The class $\left[C\left(\mathrm{P}_{1}\right)\right] \in H H_{2}^{\theta}\left(\mathbb{C}_{q}\left[U / K_{S}\right]\right)$ is non-trivial.

Proof. To show that the homology class $\left[C\left(\mathrm{P}_{1}\right)\right] \in H H_{2}^{\theta}\left(\mathbb{C}_{q}\left[U / K_{S}\right]\right)$ is non-trivial we will show that it has non-zero pairing with a cohomology class $\left[\eta_{a}\right] \in H H_{\theta}^{2}\left(\mathbb{C}_{q}\left[U / K_{S}\right]\right)$, using Proposition 3.9. Recall that for any $a \in I$ we have

$$
\left\langle\left[\eta_{a}\right],\left[C\left(\mathrm{P}_{1}\right)\right]\right\rangle=q^{\left(2 \rho-\alpha_{a}, \rho_{S}\right)}\left[\left(\alpha_{a}^{\vee}, \rho_{S}\right)\right]_{q_{a}},
$$

where we have used the fact that $\lambda_{1}=\rho_{S}$. Now for any $a \in I \backslash S$ we have $\left(\alpha_{a}^{\vee}, \rho_{S}\right)=1$, since by definition $\rho_{S}=\sum_{i \in I \backslash S} \omega_{i}$. Hence for any $a \in I \backslash S$ we obtain

$$
\left\langle\left[\eta_{a}\right],\left[C\left(\mathrm{P}_{1}\right)\right]\right\rangle=q^{\left(2 \rho-\alpha_{a}, \rho_{S}\right)} \neq 0 .
$$

From this we conclude that $\left[C\left(\mathrm{P}_{1}\right)\right]$ is non-trivial.

Thus we have shown that, for every quantum flag manifold $\mathbb{C}_{q}\left[U / K_{S}\right]$, we have a non-trivial class $\left[C\left(\mathrm{P}_{1}\right)\right] \in H H_{2}^{\theta}\left(\mathbb{C}_{q}\left[U / K_{S}\right]\right)$. These classes have an appropriate classical limit for $q \rightarrow 1$. Then, according to the Hochschild-Kostant-Rosenberg theorem, they will correspond to some differential two-forms on $U / K_{S}$. We will investigate this aspect in the following.

Recall that all flag manifolds $U / K_{S}$ are Kähler manifolds. In particular, they admit twoforms with some special properties, the Kähler forms. In the next section we will construct some Kähler forms on $U / K_{S}$ with the goal of comparing them with the classes $\left[C\left(\mathrm{P}_{1}\right)\right]$.

\section{Kähler forms on (classical) flag manifolds}

In this section we will provide a construction of some Kähler forms on the flag manifolds $U / K_{S}$. While there are many possible approaches, our aim is to proceed in a way that is well-suited for comparison with the quantum classes from the previous section. The construction will use projections analogous to those used in the quantum case. 


\subsection{Notation}

Before getting into the construction, let us quickly explain the notation we will employ. This will parallel what we have already used in the quantum case.

Recall that the Lie groups $G$ and $U$, as well as the Lie algebras $\mathfrak{g}$ and $\mathfrak{u}$, all act in a compatible way in a given finite-dimensional representation $V$. The representation of $G$ will be holomorphic, while the representation of $U$ will be unitary with respect to an appropriate inner product. We will mainly consider the algebra of matrix coefficients $\mathbb{C}[U] \subset C^{\infty}(U, \mathbb{C})$. This algebra is spanned by $c_{f, v}^{V}: U \rightarrow \mathbb{C}$ (for finite-dimensional representations) given by

$$
c_{f, v}^{V}(g):=f(g v), \quad f \in V^{*}, \quad v \in V, \quad g \in U .
$$

We will occasionally consider the matrix coefficients $c_{f, v}^{V}$ as functions on $G$ according to the same formula. In the same way we can make sense of $c_{f, v}^{V}(X)$ for $X$ in $\mathfrak{g}$ or $\mathfrak{u}$.

Now let $\left\{v_{i}\right\}_{i}$ be an orthonormal weight basis with respect to the given inner product on $V$. Let us also denote by $\left\{f^{i}\right\}_{i}$ the dual basis of $V^{*}$. Then we have $c_{f^{i}, v_{j}}^{V}(g)=\left(v_{i}, g v_{j}\right)$. Corresponding to this choice we will employ the notation $u_{j}^{i}:=c_{f^{i}, v_{j}}^{V}$, that is

$$
u_{j}^{i}(g)=\left(v_{i}, g v_{j}\right) .
$$

We will omit the index $V$, as the representation $V$ will be fixed in the following. Recall that, given a function $f: U \rightarrow \mathbb{C}$, its conjugate $\bar{f}$ is defined by $\bar{f}(g):=\overline{f(g)}$. Since the matrix with entries $u_{j}^{i}(g)$ is unitary for every $g \in U$, we obtain the identity

$$
\overline{u_{j}^{i}}(g)=u_{i}^{j}\left(g^{-1}\right)
$$

Finally let us consider the action of the Lie algebra $\mathfrak{g}$ on $c_{f, v}^{V}$. Considering $\mathfrak{g}$ as derivations at $1 \in G$, it turns out that $X\left(c_{f, v}^{V}\right)=f(X v)$, where $\mathfrak{g}$ acts on $V$ by its representation.

\subsection{The projections}

In this subsection we will construct a projection $P$ for each flag manifold $U / K_{S}$, in full analogy with the construction given in Section 4.1.

Corresponding to the subset $S \subset I$, we consider the dominant weight

$$
\rho_{S}=\sum_{i \in I \backslash S} \omega_{i}
$$

We have an irreducible representation $V\left(\rho_{S}\right)$ of highest weight $\rho_{S}$. Let $\left\{v_{i}\right\}_{i}$ be an orthonormal weight basis as above. We assume that $v_{1}$ is a highest weight vector (of weight $\rho_{S}$ ).

As we have already mentioned, the action of $P_{S}$ preserves the line $\mathbb{C} v_{1}$, see for instance [6, Section 3.2.8]. This defines a character $\xi_{\rho_{S}}: P_{S} \rightarrow \mathbb{C}^{\times}$by

$$
g v_{1}=\xi_{\rho_{S}}(g) v_{1}, \quad g \in P_{S} .
$$

This restricts to a character of $K_{S}=P_{S} \cap U$, which we denote by the same symbol.

Remark 5.1. Recall that a linear functional $\lambda \in \mathfrak{h}^{*}$ is called analytically integral (see [19, Section IV.7]) if there is a (multiplicative) character $\xi_{\lambda}: T \rightarrow \mathbb{C}^{\times}$such that

$$
\xi_{\lambda}(\exp h)=e^{\lambda(h)}, \quad \forall h \in \mathfrak{h} \cap \mathfrak{u} .
$$

Our notation $\xi_{\rho_{S}}$ for the character corresponding to $V\left(\rho_{S}\right)$ is consistent with this one. 
Now using the unitary matrix coefficients $u_{j}^{i}$ of $V\left(\rho_{S}\right)$ we define

$$
p_{j}^{i}:=u_{1}^{i} \overline{u_{1}^{j}} \in \mathbb{C}[U] \subset C^{\infty}(U, \mathbb{C}) .
$$

We will now derive some properties satisfied by the functions $p_{j}^{i}$.

Lemma 5.2. We have $p_{j}^{i} \in C^{\infty}\left(U / K_{S}, \mathbb{C}\right)$. Moreover we have the identities

$$
\sum_{k} p_{k}^{i} p_{j}^{k}=p_{j}^{i}, \quad \overline{p_{j}^{i}}=p_{i}^{j}, \quad \sum_{i} p_{i}^{i}=1 .
$$

Proof. We need to show that $p_{j}^{i}(g k)=p_{j}^{i}(g)$ for all $g \in U$ and $k \in K_{S}$. We compute

$$
u_{1}^{i}(g k)=\left(v_{i}, g k v_{1}\right)=\xi_{\rho_{S}}(k) u_{1}^{i}(g) .
$$

On the other hand, using $\overline{u_{1}^{j}}(g)=u_{j}^{1}\left(g^{-1}\right)$ we compute

$$
\overline{u_{1}^{j}}(g k)=u_{j}^{1}\left(k^{-1} g^{-1}\right)=\left(v_{1}, k^{-1} g^{-1} v_{j}\right)=\left(k v_{1}, g^{-1} v_{j}\right)=\overline{\xi_{\rho_{S}}(k)} \overline{u_{1}^{j}}(g) .
$$

Here we have used that the representation is unitary. Therefore

$$
p_{j}^{i}(g k)=u_{1}^{i}(g k) \overline{u_{1}^{j}}(g k)=\left|\xi_{\rho_{S}}(k)\right|^{2} u_{1}^{i}(g) \overline{u_{1}^{j}}(g)=p_{j}^{i}(g) .
$$

The other properties are easy to check.

We denote by $P$ the matrix with entries $p_{j}^{i}$. By the previous lemma, this is an orthogonal projection of rank 1 with entries in $C^{\infty}\left(U / K_{S}, \mathbb{C}\right)$.

\subsection{Line bundles}

In this subsection we will interpret the functions $u_{1}^{i}$ as sections of a line bundle over $G / P_{S}$ $\cong U / K_{S}$. The material here will be used only tangentially in the following, but it gives an interesting geometric perspective, as well as connecting the construction we will use with other ways to obtain Kähler forms on flag manifolds.

Recall that the Lie group $G$ can be considered as a principal $P_{S}$-bundle over the flag manifold $G / P_{S}$ (similarly for the compact description $U / K_{S}$ ). Given a representation of $G$ on a vector space $V$, we can form the associated vector bundle $G \times{ }_{P_{S}} V$. The points of this bundle are the equivalence classes of $G \times V$ with respect to the relation

$$
(g, v) \sim\left(g p, p^{-1} v\right), \quad g \in G, \quad p \in P_{S}, \quad v \in V .
$$

This is a holomorphic vector bundle if the given representation is holomorphic. The sections of this bundle can be identified with the functions $f: G \rightarrow V$ such that

$$
f(g p)=p^{-1} f(g), \quad g \in G, \quad p \in P_{S} .
$$

Now let us consider the holomorphic line bundle

$$
L_{-\rho_{S}}:=G \times{ }_{P_{S}} \mathbb{C}
$$

where $P_{S}$ acts on $\mathbb{C}$ by $p \cdot z=\xi_{\rho_{S}}(p)^{-1} z$. Observe that this is a holomorphic representation, since the character $\xi_{S}$ comes from the holomorphic representation of $G$ on $V\left(\rho_{S}\right)$.

Lemma 5.3. The functions $u_{1}^{i}: G \rightarrow \mathbb{C}$ are holomorphic sections of $L_{-\rho_{S}}$. 
Proof. It is clear that they are holomorphic functions, since $u_{1}^{i}(g)=\left(v_{i}, g v_{1}\right)$ for $g \in G$ and the representation of $G$ on $V\left(\rho_{S}\right)$ is holomorphic. To show that they are sections of $L_{-\rho_{S}}$ we observe that $p v_{1}=\xi_{\rho_{S}}(p) v_{1}$ implies $u_{1}^{i}(g p)=\xi_{\rho_{S}}(p) u_{1}^{i}(g)$ for $p \in P_{S}$.

Remark 5.4. The minus sign in the definition of $L_{-\rho_{S}}$ (that is, using the character $\xi_{\rho_{S}}^{-1}$ instead of $\left.\xi_{\rho_{S}}\right)$ is due to the fact that we take the positive Borel subgroup, as opposed to the negative one, which is the more common choice when stating the Borel-Weil theorem. For a formulation using this convention see for instance [26, Theorem 7.58].

It is possible to proceed along these lines to obtain a Kähler form on $U / K_{S}$, as we will now sketch. Equipping the line bundle $L_{-\rho_{S}}$ with a connection, its curvature gives a $(1,1)$-form $\eta$ (a representative of the first Chern class of $U / K_{S}$ ). Then $\eta$ is Kähler if the line bundle $L_{-\rho_{S}}$ is positive, which in turn is equivalent to $L_{-\rho_{S}}$ being ample by Kodaira's embedding theorem. But it is known that $L_{-\rho_{S}}$ is ample by results of Borel-Weil, see for instance [27, Theorem 6.5] (keeping in mind the opposite convention for the Borel subgroup).

However we will not proceed this way, since this description is not particularly well-suited for comparison with the quantum setting. Instead, we will define the candidate Kähler form in terms of the projection $P$ introduced before, using the Chern character.

\subsection{Differential forms}

Let $P$ be the projection with entries $p_{j}^{i} \in C^{\infty}\left(U / K_{S}, \mathbb{C}\right)$ from (5.1). Corresponding to this projection, we define a two-form on $U / K_{S}$ by

$$
\tilde{\omega}:=\operatorname{Tr}(P \cdot \mathrm{d} P \wedge \mathrm{d} P)=\sum_{i, j, k} p_{j}^{i} \mathrm{~d} p_{k}^{j} \wedge \mathrm{d} p_{i}^{k} .
$$

Here and in the following we will adopt some obvious matrix-type notation. Our aim will be to show that $\tilde{\omega}$ is, up to a constant, a Kähler form on $U / K_{S}$.

Before getting into that, let us motivate this choice from a suitably non-commutative point of view. By Lemma 5.2 the matrix $P$ with entries $p_{j}^{i} \in C^{\infty}\left(U / K_{S}, \mathbb{C}\right)$ is a projection of rank one. Hence we have a projective $C^{\infty}\left(U / K_{S}, \mathbb{C}\right)$-module of rank one which, according to the SerreSwan theorem, corresponds to a complex line bundle over $U / K_{S}$. Moreover this bundle admits a Hermitian structure, due to the fact that $P$ is orthogonal. More importantly, this line bundle admits a natural connection defined in terms of the projection $P$, namely the Levi-Civita one. Its curvature can be computed using the Chern character and coincides with the two-form $\tilde{\omega}$ defined above, up to a factor. For more on this point of view, see for instance [17, Chapter 1] and [21, Chapter 8].

We will now show some basic properties of $\tilde{\omega}$. We remark that the fact that it is closed is a general result of Chern-Weil theory, but we give a short proof for completeness.

Lemma 5.5. The two-form $\tilde{\omega}$ on $U / K_{S}$ satisfies the properties:

1) it is closed,

2) it is left $U$-invariant.

Proof. (1) The exterior derivative of $\tilde{\omega}$ is the 3 -form given by

$$
\mathrm{d} \tilde{\omega}=\sum_{i, j, k} \mathrm{~d} p_{j}^{i} \wedge \mathrm{d} p_{k}^{j} \wedge \mathrm{d} p_{i}^{k}=\operatorname{Tr}(\mathrm{d} P \wedge \mathrm{d} P \wedge \mathrm{d} P) .
$$

Now consider the element $J=2 P-1$, which satisfies $J^{2}=1$. Applying d to this identity we get $J \mathrm{~d} P=-\mathrm{d} P J$. Using these properties of $J$, we can observe that

$$
\mathrm{d} \tilde{\omega}=\operatorname{Tr}\left(J^{2} \mathrm{~d} P \wedge \mathrm{d} P \wedge \mathrm{d} P\right)=-\operatorname{Tr}(J \mathrm{~d} P \wedge \mathrm{d} P \wedge \mathrm{d} P J)=-\operatorname{Tr}(\mathrm{d} P \wedge \mathrm{d} P \wedge \mathrm{d} P)=0 .
$$


(2) The left translation $L_{g}: U \rightarrow U$ given by $L_{g} h=g h$ induces a map $L_{g}: U / K_{S} \rightarrow U / K_{S}$, denoted by the same symbol. A form $\omega$ on $U / K_{S}$ is left $U$-invariant if $L_{g}^{*} \omega=\omega$ for every $g \in U$. In other words, for any $g \in U$ we must have $L_{g}^{*} \omega_{g h}=\omega_{h}$ for every $h \in U / K_{S}$. For the matrix coefficients $u_{j}^{i}$, considered as functions on $U$, we have

$$
L_{g}^{*} u_{j}^{i}=\sum_{k} \pi(g)_{k}^{i} u_{j}^{k}, \quad L_{g}^{*} \overline{u_{j}^{i}}=\sum_{k} \pi\left(g^{-1}\right)_{i}^{k} \overline{u_{j}^{k}} .
$$

Here $\pi$ denotes the representation of $U$ on $V\left(\rho_{S}\right)$. From these identities we immediately obtain that the pullback of the functions $p_{j}^{i}$ is given by

$$
L_{g}^{*} p_{j}^{i}=\sum_{k, l} \pi(g)_{k}^{i} \pi\left(g^{-1}\right)_{j}^{l} p_{l}^{k} .
$$

Using this fact, it is easy to check that we have the identity

$$
\sum_{i, j, k} L_{g}^{*} p_{j}^{i} \otimes L_{g}^{*} p_{k}^{j} \otimes L_{g}^{*} p_{i}^{k}=\sum_{i, j, k} p_{j}^{i} \otimes p_{k}^{j} \otimes p_{i}^{k} .
$$

Since pullbacks are compatible with the wedge product and commute with the exterior derivative, we conclude that $L_{g}^{*} \tilde{\omega}_{g h}=\tilde{\omega}_{h}$ and hence $\tilde{\omega}$ is left $U$-invariant.

\subsection{Complex decomposition}

In this section we explore the consequences of the $u_{1}^{i}$ being holomorphic sections of a line bundle over $U / K_{S}$. We begin with a simple lemma.

Lemma 5.6. We have the identities

$$
P \cdot \partial P=0, \quad \partial P \cdot P=\partial P, \quad P \cdot \bar{\partial} P=\bar{\partial} P, \quad \bar{\partial} P \cdot P=0 .
$$

Proof. These can be easily derived from Lemma 5.2 together with $\bar{\partial}\left(u_{1}^{i}\right)=0$ and $\partial\left(\overline{u_{1}^{j}}\right)=0$, where the last two identities follow from $u_{1}^{i}$ being holomorphic. For instance we have

$$
\partial p_{j}^{i}=\left(\partial u_{1}^{i}\right) \overline{u_{1}^{j}}=\sum_{k}\left(\partial u_{1}^{i}\right) \overline{u_{1}^{k}} u_{1}^{k} \overline{u_{1}^{j}}=\sum_{k}\left(\partial p_{k}^{i}\right) p_{j}^{k} .
$$

Remark 5.7. There is an exact analogue of these identities for the quantum irreducible flag manifolds in terms of the Heckenberger-Kolb calculus, see [22, Lemma 5.2].

We will now rescale $\tilde{\omega}$ by setting

$$
\omega:=-i \tilde{\omega}=-i \operatorname{Tr}(P \cdot \mathrm{d} P \wedge \mathrm{d} P) .
$$

We recall that $\boldsymbol{i} \in \mathbb{C}$ denotes the imaginary unit. The main reason for this rescaling is to make $\omega$ into a real form (and also positive definite, as we will see later on).

Lemma 5.8. We have that $\omega$ is a real $(1,1)$-form. Moreover

$$
\omega=\boldsymbol{i} \operatorname{Tr}(\partial P \wedge \bar{\partial} P)=i \sum_{i, j} \partial p_{j}^{i} \wedge \bar{\partial} p_{i}^{j} .
$$

Proof. Taking into account the identities from Lemma 5.6 we rewrite

$$
\begin{aligned}
\tilde{\omega} & =\operatorname{Tr}(P \cdot(\partial P+\bar{\partial} P) \wedge \mathrm{d} P)=\operatorname{Tr}(\bar{\partial} P \wedge \mathrm{d} P)=\operatorname{Tr}(\bar{\partial} P \wedge \partial P)+\operatorname{Tr}(\bar{\partial} P \wedge \bar{\partial} P) \\
& =\operatorname{Tr}(\bar{\partial} P \wedge \partial P) .
\end{aligned}
$$

Since $\omega=-\boldsymbol{i} \tilde{\omega}$, we conclude that $\omega$ is a $(1,1)$-form with the claimed expression. Next we show that $\omega$ is real, that is $\bar{\omega}=\omega$. Using the identity $\overline{p_{j}^{i}}=p_{i}^{j}$ we compute

$$
\bar{\omega}=-i \sum_{i, j} \overline{\partial p_{j}^{i}} \wedge \overline{\bar{\partial}} p_{i}^{j}=-i \sum_{i, j} \bar{\partial} p_{i}^{j} \wedge \partial p_{j}^{i}=\omega .
$$




\subsection{Kähler forms}

The notion of Kähler form on a complex manifold is a standard one. The most convenient characterization for us is the following (as in [16, Lemma 3.1.7], for instance): a Kähler form on $X$ is a closed real $(1,1)$-form $\omega$ which is positive definite, that is locally of the form $\omega=$ $\frac{i}{2} \sum_{i, j} h_{i j} \mathrm{~d} z_{i} \wedge \mathrm{d} \bar{z}_{j}$ with $h_{i j}(x)$ a positive definite Hermitian matrix for all $x \in X$. This is strictly related to the notion of Kähler metric on $X$.

Our goal is to show that $\omega$ from (5.2) is a Kähler form on the flag manifold $U / K_{S}$. So far we have shown that it is a closed real $(1,1)$-form on $U / K_{S}$, which is also $U$-invariant. To conclude that it is a Kähler form we still need to show that it is positive definite.

As $\omega$ is $U$-invariant, it suffices to show that it is positive definite at the origin $o:=K_{S}$, namely the identity coset of $U / K_{S}$. Recall that, since $U / K_{S} \cong G / P_{S}$, we can identify the holomorphic tangent space at $o$ with $\mathfrak{g} / \mathfrak{p}_{S} \cong \mathfrak{n}_{S}^{-}$and the anti-holomorphic tangent space with $\mathfrak{n}_{S}^{+}$. In other words, the former is the span of the root vectors $\left\{f_{\alpha}\right\}_{\alpha \in \Delta\left(\mathfrak{n}_{S}^{+}\right)}$, while the latter is the span of the root vectors $\left\{e_{\alpha}\right\}_{\alpha \in \Delta\left(\mathfrak{n}_{S}^{+}\right)}$. We will denote by $f_{\alpha}^{\star}$ and $e_{\alpha}^{\star}$ the corresponding dual elements in their respective cotangent spaces.

From the above discussion and the fact that $\omega$ is a $(1,1)$-form, it follows that it must take the following form at the origin

$$
\omega_{o}=i \sum_{\alpha, \beta \in \Delta\left(\mathfrak{n}_{S}^{+}\right)} c_{\alpha \beta} f_{\alpha}^{\star} \wedge e_{\beta}^{\star}, \quad c_{\alpha \beta} \in \mathbb{C} .
$$

To show that $\omega$ is positive definite it suffices to show that the matrix with entries $c_{\alpha \beta}$ is positive definite. This is what we will check in the following.

First we will require the following simple lemma.

Lemma 5.9. Let $\alpha \in \Delta\left(\mathfrak{n}_{S}^{+}\right)$. Then $v_{\alpha}=\left(\rho_{S}, \alpha\right)^{-1 / 2} f_{\alpha} v_{1}$ is a vector of norm 1 .

Proof. Recall that $v_{1}$ is a highest weight vector of weight $\rho_{S}$. Hence we have

$$
e_{\alpha} f_{\alpha} v_{1}=\left[e_{\alpha}, f_{\alpha}\right] v_{1}=h_{\alpha} v_{1}=\left(\rho_{S}, \alpha\right) v_{1} .
$$

Now we observe that, since $\rho_{S}=\sum_{i \in I \backslash S} \omega_{i}$ and $\alpha \in \Delta\left(\mathfrak{n}_{S}^{+}\right)$, we have $\left(\rho_{S}, \alpha\right)>0$. Hence we can consider the vector $v_{\alpha}=\left(\rho_{S}, \alpha\right)^{-1 / 2} f_{\alpha} v_{1}$. To show that it has norm 1 we compute

$$
\left(v_{\alpha}, v_{\alpha}\right)=\left(\rho_{S}, \alpha\right)^{-1}\left(f_{\alpha} v_{1}, f_{\alpha} v_{1}\right)=\left(\rho_{S}, \alpha\right)^{-1}\left(v_{1}, e_{\alpha} f_{\alpha} v_{1}\right)=1 .
$$

Up to now the choice of the orthonormal weight basis $\left\{v_{i}\right\}_{i}$ for $V\left(\rho_{S}\right)$ was arbitrary. We will now require that the basis contains all the vectors $v_{\alpha}$ for $\alpha \in \Delta\left(\mathfrak{n}_{S}^{+}\right)$as in the lemma above. Notice that this requirement makes sense, as they all have norm 1.

Theorem 5.10. We have that $\omega$ is a Kähler form on $U / K_{S}$. Moreover

$$
\omega_{o}=\boldsymbol{i} \sum_{\alpha \in \Delta\left(\mathfrak{n}_{S}^{+}\right)}\left(\rho_{S}, \alpha\right) f_{\alpha}^{\star} \wedge e_{\alpha}^{\star}
$$

Proof. We have already shown that $\omega$ is a closed, real $(1,1)$-form. Hence it suffices to show that $\omega$ is positive definite (that is, the corresponding symmetric bilinear form is positive definite). As $\omega$ is $U$-invariant, it suffices to show this at the origin of $U / K_{S}$. As we have already discussed, the $(1,1)$-form $\omega$ at the origin has the following form

$$
\omega_{o}=i \sum_{\alpha, \beta \in \Delta\left(\mathfrak{n}_{S}^{+}\right)} c_{\alpha \beta} f_{\alpha}^{\star} \wedge e_{\beta}^{\star} .
$$


To show that $\omega$ is positive definite it suffices to show that the matrix with entries $c_{\alpha \beta}$ is positive definite. Observe that $c_{\alpha \beta}=-i \omega_{o}\left(f_{\alpha}, e_{\beta}\right)$.

We will now determine $c_{\alpha \beta}$ using the expression $\omega=-i \sum_{i, j, k} p_{j}^{i} \mathrm{~d} p_{k}^{j} \wedge \mathrm{d} p_{i}^{k}$. We will consider $o=K_{S}$ as being $1 \in U$, as the result will not depend on the chosen representative. Then

$$
c_{\alpha \beta}=-\sum_{i, j, k} p_{j}^{i}(1) f_{\alpha}\left(p_{k}^{j}\right) e_{\beta}\left(p_{i}^{k}\right)=-\sum_{i, j} f_{\alpha}\left(p_{j}^{i}\right) e_{\beta}\left(p_{i}^{j}\right) .
$$

In the last step we have used that $p_{j}^{i}(1)=\delta_{j}^{i}$, which follows from $p_{j}^{i}(1)=\left(v_{i}, v_{j}\right)$. Moreover, since $p_{j}^{i}=u_{1}^{i} \overline{u_{1}^{j}}$ and $f_{\alpha}$ is a derivation at the identity, we have

$$
f_{\alpha}\left(p_{j}^{i}\right)=f_{\alpha}\left(u_{1}^{i}\right) \overline{u_{1}^{j}}(1)+u_{1}^{i}(1) f_{\alpha}\left(\overline{u_{1}^{j}}\right)=\delta_{1}^{j} f_{\alpha}\left(u_{1}^{i}\right) .
$$

Here we have used the fact that $f_{\alpha}\left(\overline{u_{1}^{j}}\right)=0$ by weight reasons. Similar computations show that $e_{\beta}\left(p_{i}^{j}\right)=\delta_{1}^{j} e_{\beta}\left(\overline{u_{1}^{i}}\right)$. Therefore we obtain the expression

$$
c_{\alpha \beta}=-\sum_{i} f_{\alpha}\left(u_{1}^{i}\right) e_{\beta}\left(\overline{u_{1}^{i}}\right) .
$$

First we will consider $f_{\alpha}\left(u_{1}^{i}\right)=\left(v_{i}, f_{\alpha} v_{1}\right)$. Since $f_{\alpha} v_{1}$ is proportional to $v_{\alpha}$, it is clear that this is zero unless $v_{i}=v_{\alpha}$ (as the chosen basis is orthonormal). In this case we have

$$
f_{\alpha}\left(u_{1}^{i}\right)=\left(v_{\alpha}, f_{\alpha} v_{1}\right)=\left(\rho_{S}, \alpha\right)^{1 / 2}\left(v_{\alpha}, v_{\alpha}\right)=\left(\rho_{S}, \alpha\right)^{1 / 2} .
$$

Similarly consider $e_{\beta}\left(\overline{u_{1}^{i}}\right)=-\overline{\left(v_{i}, f_{\beta} v_{1}\right)}$. Again this is zero unless $v_{i}=v_{\alpha}$, in which case $e_{\beta}\left(\overline{u_{1}^{i}}\right)=-\left(\rho_{S}, \alpha\right)^{1 / 2}$. Hence we conclude that $c_{\alpha \beta}=0$ for $\alpha \neq \beta$, while

$$
c_{\alpha \alpha}=\left(\rho_{S}, \alpha\right), \quad \alpha \in \Delta\left(\mathfrak{n}_{S}^{+}\right) .
$$

Since $\left(\rho_{S}, \alpha\right)>0$ for $\alpha \in \Delta\left(\mathfrak{n}_{S}^{+}\right)$, we conclude that $\omega$ is positive definite.

Remark 5.11. This result is essentially due to Borel-Hirzebruch, [4, Proposition 14.6]. See also [27, Theorem 7.5], taking into account different conventions.

\section{Comparison in the classical limit}

In this last section we will consider an appropriate classical limit of the classes $\left[C\left(\mathrm{P}_{1}\right)\right] \in$ $H H_{2}^{\theta}\left(\mathbb{C}_{q}\left[U / K_{S}\right]\right)$ constructed in Section 3. We will show that the corresponding classical twoforms can be identified with the Kähler forms on $U / K_{S}$ constructed in Section 5 .

\subsection{Remarks on the classical limit}

Informally, the quantized enveloping algebra $U_{q}(\mathfrak{u})$ reduces to the enveloping algebra $U(\mathfrak{u})$ for $q \rightarrow 1$, which we refer to as the classical limit. This specialization can be made precise with a bit of care, see for instance the approach in [15, Section 3.4]. For our purposes, the only thing we need to know is that the classical limit $q \rightarrow 1$ makes sense at the level of representations.

Next, we want to consider the classical limit of the quantized coordinate ring $\mathbb{C}_{q}[U]$. This was defined as the algebra of matrix coefficients of finite-dimensional $U_{q}(\mathfrak{u})$-representations, hence for $q \rightarrow 1$ it reduces to the algebra of matrix coefficients of finite-dimensional $U(\mathfrak{u})$-representations. Finally we can identify this algebra with $\mathbb{C}[U] \subset C^{\infty}(U, \mathbb{C})$, the algebra of matrix coefficients of finite-dimensional $U$-representations. This is simply done by

$$
c_{f, v}^{V}(X)=f(X v) \longleftrightarrow c_{f, v}^{V}(g)=f(g v),
$$

where $X \in U(\mathfrak{u})$ and $g \in U$. Observe that this identification makes sense, since $U$ and $\mathfrak{u}$ act in a compatible way in each finite-dimensional representation $V$. 


\subsection{The comparison}

Consider now the representative $C\left(\mathrm{P}_{1}\right) \in \mathbb{C}_{q}\left[U / K_{S}\right]^{\otimes 3}$ of the class $\left[C\left(\mathrm{P}_{1}\right)\right] \in H H_{2}^{\theta}\left(\mathbb{C}_{q}\left[U / K_{S}\right]\right)$. It corresponds to an element $\widetilde{C\left(\mathrm{P}_{1}\right)} \in \mathbb{C}\left[U / K_{S}\right]^{\otimes 3}$ under the classical limit, as explained above. Denote by $\omega_{\mathrm{P}_{1}} \in \Omega^{2}\left(U / K_{S}\right)$ the corresponding two-form obtained from the Hochschild-KostantRosenberg map.

Theorem 6.1. The (rescaled) form $\omega_{\mathrm{P}_{1}} \in \Omega^{2}\left(U / K_{S}\right)$ is a Kähler form.

Proof. The representative $C\left(\mathrm{P}_{1}\right) \in \mathbb{C}_{q}\left[U / K_{S}\right]^{\otimes 3}$ is given explicitly by

$$
C\left(\mathrm{P}_{1}\right)=\sum_{i, j, k} q^{\left(2 \rho, \lambda_{i}\right)}\left(2 p_{j}^{i}-\delta_{j}^{i}\right) \otimes p_{k}^{j} \otimes p_{i}^{k}
$$

The elements $p_{j}^{i} \in \mathbb{C}_{q}[U]$ from (4.1) correspond to the elements $p_{j}^{i} \in \mathbb{C}[U]$ from (5.1) under the classical limit. Hence we obtain

$$
\widetilde{C\left(\mathrm{P}_{1}\right)}=\sum_{i, j, k}\left(2 p_{j}^{i}-\delta_{j}^{i}\right) \otimes p_{k}^{j} \otimes p_{i}^{k} \in \mathbb{C}\left[U / K_{S}\right]^{\otimes 3} .
$$

Now $\omega_{\mathrm{P}_{1}}$ is the image of $\widetilde{C\left(\mathrm{P}_{1}\right)}$ under the Hochschild-Kostant-Rosenberg map, which is given by $a_{0} \otimes a_{1} \otimes \cdots a_{n} \mapsto a_{0} \mathrm{~d} a_{1} \wedge \cdots \wedge \mathrm{d} a_{n}$. We obtain the two-form

$$
\omega_{\mathrm{P}_{1}}=\sum_{i, j, k}\left(2 p_{j}^{i}-\delta_{j}^{i}\right) \mathrm{d} p_{k}^{j} \wedge \mathrm{d} p_{i}^{k} \in \Omega^{2}\left(U / K_{S}\right) .
$$

Observe that the second term is zero by graded-commutativity, since

$$
\sum_{i, j} \mathrm{~d} p_{j}^{i} \wedge \mathrm{d} p_{i}^{j}=-\sum_{i, j} \mathrm{~d} p_{i}^{j} \wedge \mathrm{d} p_{j}^{i}=0
$$

Hence $\omega_{\mathrm{P}_{1}}$ can be rewritten as

$$
\omega_{\mathrm{P}_{1}}=2 \sum_{i, j, k} p_{j}^{i} \mathrm{~d} p_{k}^{j} \wedge \mathrm{d} p_{i}^{k} \in \Omega^{2}\left(U / K_{S}\right)
$$

This coincides with $\omega$ as defined in (5.2), up to a constant. But we know that the latter is a Kähler form on $U / K_{S}$ according to Theorem 5.10, which gives the claim.

Remark 6.2. For a quantum irreducible flag manifold $\mathbb{C}_{q}\left[U / K_{S}\right]$, we have constructed in [22] a Kähler form in the sense of [25] using the differential calculus of [13]. It is of the form $\sum_{i, j, k} q^{\left(2 \rho, \lambda_{i}\right)} p_{j}^{i} \mathrm{~d} p_{k}^{j} \wedge p_{i}^{k}$, up to a constant. Hence, by the same argument as above, it can be identified with a Kähler form on the irreducible flag manifold $U / K_{S}$.

To summarize, the non-trivial classes $\left[C\left(\mathrm{P}_{1}\right)\right] \in H H_{2}^{\theta}\left(\mathbb{C}_{q}\left[U / K_{S}\right]\right)$ constructed in this paper correspond to some Kähler forms on $U / K_{S}$, under an appropriate classical limit. For this reason we believe they will play an important role in the investigation of the non-commutative complex and Kähler geometry of the quantum flag manifolds $\mathbb{C}_{q}\left[U / K_{S}\right]$.

\section{References}

[1] Akrami S.E., Majid S., Braided cyclic cocycles and nonassociative geometry, J. Math. Phys. 45 (2004), 3883-3911, arXiv:math.QA/0406005.

[2] Baez J.C., Hochschild homology in a braided tensor category, Trans. Amer. Math. Soc. 344 (1994), 885-906. 
[3] Beggs E., Paul Smith S., Non-commutative complex differential geometry, J. Geom. Phys. 72 (2013), 7-33, arXiv:1209.3595.

[4] Borel A., Hirzebruch F., Characteristic classes and homogeneous spaces. I, Amer. J. Math. 80 (1958), 458-538.

[5] Brown K.A., Zhang J.J., Dualising complexes and twisted Hochschild (co)homology for Noetherian Hopf algebras, J. Algebra 320 (2008), 1814-1850, arXiv:math.RA/0603732.

[6] Čap A., Slovák J., Parabolic geometries. I. Background and general theory, Mathematical Surveys and Monographs, Vol. 154, Amer. Math. Soc., Providence, RI, 2009.

[7] Connes A., Noncommutative differential geometry, Inst. Hautes Études Sci. Publ. Math. (1985), 41-144.

[8] Dolgushev V., The Van den Bergh duality and the modular symmetry of a Poisson variety, Selecta Math. (N.S.) 14 (2009), 199-228, arXiv:math.QA/0612288.

[9] Feng P., Tsygan B., Hochschild and cyclic homology of quantum groups, Comm. Math. Phys. 140 (1991), 481-521.

[10] Hadfield T., Twisted cyclic homology of all Podleś quantum spheres, J. Geom. Phys. 57 (2007), 339-351, arXiv:math.QA/0405243.

[11] Hadfield T., Krähmer U., Twisted homology of quantum SL(2), K-Theory 34 (2005), 327-360, arXiv:math.QA/0405249.

[12] Hadfield T., Krähmer U., Braided homology of quantum groups, J. K-Theory 4 (2009), 299-332, arXiv:math.QA/0701193.

[13] Heckenberger I., Kolb S., De Rham complex for quantized irreducible flag manifolds, J. Algebra 305 (2006), 704-741, arXiv:math.QA/0307402.

[14] Hochschild G., Kostant B., Rosenberg A., Differential forms on regular affine algebras, Trans. Amer. Math. Soc. 102 (1962), 383-408.

[15] Hong J., Kang S.J., Introduction to quantum groups and crystal bases, Graduate Studies in Mathematics, Vol. 42, Amer. Math. Soc., Providence, RI, 2002.

[16] Huybrechts D., Complex geometry: an introduction, Universitext, Springer-Verlag, Berlin, 2005.

[17] Karoubi M., Homologie cyclique et K-théorie, Astérisque 149 (1987), 147 pages.

[18] Klimyk A., Schmüdgen K., Quantum groups and their representations, Texts and Monographs in Physics, Springer-Verlag, Berlin, 1997.

[19] Knapp A.W., Lie groups beyond an introduction, Progress in Mathematics, Vol. 140, Birkhäuser Boston, Inc., Boston, MA, 1996.

[20] Kustermans J., Murphy G.J., Tuset L., Differential calculi over quantum groups and twisted cyclic cocycles, J. Geom. Phys. 44 (2003), 570-594, arXiv:math.QA/0110199.

[21] Loday J.-L., Cyclic homology, 2nd ed., Grundlehren der Mathematischen Wissenschaften, Vol. 301, SpringerVerlag, Berlin, 1998.

[22] Matassa M., Kähler structures on quantum irreducible flag manifolds, J. Geom. Phys. 145 (2019), 103477, 16 pages, arXiv:1901.09544.

[23] Matassa M., Twisted Hochschild homology of quantum flag manifolds: 2-cycles from invariant projections, J. Algebra Appl., to appear, arXiv:1703.00725.

[24] Ó Buachalla R., Noncommutative complex structures on quantum homogeneous spaces, J. Geom. Phys. 99 (2016), 154-173, arXiv:1108.2374.

[25] Ó Buachalla R., Noncommutative Kähler structures on quantum homogeneous spaces, Adv. Math. 322 (2017), 892-939, arXiv:1602.08484.

[26] Sepanski M.R., Compact Lie groups, Graduate Texts in Mathematics, Vol. 235, Springer, New York, 2007.

[27] Snow D.M., Homogeneous vector bundles, in Group Actions and Invariant Theory (Montreal, PQ, 1988), CMS Conf. Proc., Vol. 10, Amer. Math. Soc., Providence, RI, 1989, 193-205.

[28] Stokman J.V., Dijkhuizen M.S., Quantized flag manifolds and irreducible *-representations, Comm. Math. Phys. 203 (1999), 297-324, arXiv:math.QA/9802086.

[29] van den Bergh M., A relation between Hochschild homology and cohomology for Gorenstein rings, Proc. Amer. Math. Soc. 126 (1998), 1345-1348.

[30] Woronowicz S.L., Differential calculus on compact matrix pseudogroups (quantum groups), Comm. Math. Phys. 122 (1989), 125-170. 